This item was submitted to Loughborough's Research Repository by the author.

Items in Figshare are protected by copyright, with all rights reserved, unless otherwise indicated.

\title{
Ending the vicious cycle of patient falls
}

PLEASE CITE THE PUBLISHED VERSION

http://dx.doi.org/10.1177/2327857914031021

\section{PUBLISHER}

(c) Human Factors and Ergonomics Society. Published by Sage Journals

\section{VERSION}

AM (Accepted Manuscript)

\section{PUBLISHER STATEMENT}

This work is made available according to the conditions of the Creative Commons Attribution-NonCommercialNoDerivatives 4.0 International (CC BY-NC-ND 4.0) licence. Full details of this licence are available at: https://creativecommons.org/licenses/by-nc-nd/4.0/

\section{LICENCE}

CC BY-NC-ND 4.0

\section{REPOSITORY RECORD}

Wolf, Laurie, Eileen Constantinou, and Sue Hignett. 2019. "Ending the Vicious Cycle of Patient Falls". figshare. https://hdl.handle.net/2134/25394. 


\title{
ENDING THE VICIOUS CYCLE OF PATIENT FALLS...
}

\author{
Laurie Wolf ${ }^{1,2}$, Eileen Costantinou ${ }^{2}$, Sue Hignett ${ }^{1}$ \\ ${ }^{1}$ Design School, Loughborough University. UK, ${ }^{2}$ Barnes-Jewish Hospital, St Louis, MO
}

\begin{abstract}
Over the past two years Barnes-Jewish Hospital has used Lean and Six Sigma methodologies in process improvement projects to prevent inpatient falls and falls with injury. These intensive programs have validated that falls are a multifaceted, complex problem that need constant vigilance and continuous improvement to sustain patient safety. Falls that result in serious injury can be life-changing for patients and families as well as impact the caregivers with potentially severe financial and health consequences. Trends in fall rates after completion of two Case Studies show that while decreasing the number of falls continue to be a challenge; the severity of injury from a fall can be reduced with patient and staff collaboration.
\end{abstract}

\section{INTRODUCTION}

\section{METHOD}

\section{Purpose}

Despite years of dedicated effort to keep patients safe, last year Barnes-Jewish Hospital (BJH) had 1,220 falls in which 15 resulted in a severe injury. A previous study revealed a patient that experiences a severe injury from a fall at BJC has greater operational cost of $\$ 13,316$ and stayed 6.3 days longer than a patient that did not fall (Wong et al., 2011). BJH is a member of BJC Healthcare and is affiliated with Washington University. The following two case studies were conducted at this large urban hospital in St. Louis, $\mathrm{MO}$ as part of a $\mathrm{PhD}$ program with Loughborough University.

\section{Background}

Preventing patients from being injured due to a fall during their hospitalization has been a concern in healthcare for many years. Organizations around the world such as Institute of Medicine, The Joint Commission, National Institute for Health and Clinical Excellence, National Australian Patient Safety Foundation, and the World Health Organization have been conducting research and publishing guidelines to identify evidence based interventions for fall prevention (Di Pilla \& Di Pilla, 2010; Ulrich et al., 2008). Falls are the most common cause of non-fatal injury and hospital admission for trauma. Death rates due to falls have risen sharply over the past decade due to aging of the population.
The PhD work discussed in this paper involves two case studies conducted on the same Oncology division to investigate the use of Lean and Six Sigma to prevent inpatient falls and injury. Notes taken throughout these studies have been assembled into insights for future fall prevention interventions.

\section{Case Study \#1: Lean Methodology}

Objective. The objective of Case Study \#1 was to investigate the use of Lean methodology to reduce falls with injury as part of a system-wide initiative. By standardizing assessment, intervention and post-fall investigation processes, the goal was to decrease patient falls and falls with injury rates by $50 \%$ and $30 \%$ respectively.

Method. Barnes-Jewish Hospital participated with Center for Clinical Excellence (CCE) in a Rapid Improvement Event (RIE) on three oncology divisions as part of the preventable harm initiative. Three oncology divisions were selected because they were among the highest fall with injury rate divisions in the healthcare system. For this patient population it is important to include the family in the intervention because patients can be confused and overwhelmed physically and emotionally from their illness and treatment.

A 3-day event was conducted to implement the fall prevention initiative because it aligned with the hospital's lean transformation initiative. Collaboration with various departmental staff (physical and occupational therapy, pharmacy, 
physicians, information systems, low bed equipment vendors, and clinical operations) was required to achieve multidisciplinary input. The Lean technique of standard work was used to improve fall risk assessment and intervention selection.

By the end of the RIE the team had developed standard methods to select interventions based on fall risk assessments and established a process to collect information after a fall and post it on a fall tracker board to achieve transparency of trends.(Wolf, Costantinou, Matt, \& Schulte, 2013).

Case Study \#1 Results. A 22\% decrease in total fall rate and a $37 \%$ decrease in falls with injury rate were achieved in the 16-month post-intervention period. For total falls the difference from baseline to post-RIE (5.93/1,000 patient days versus 4.1/1,000 patient days respectively) was statistically significant $(\mathrm{p}<0.05)$. Although a $22 \%$ decrease in total falls did not meet the goal of $50 \%$ decrease, the total falls with injury decrease of $37 \%$ did exceed the goal of 30\%. (Wolf, et al 2013) However one of these divisions still had one of the highest falls with injury rates in the entire BJH Healthcare system, so additional improvement was needed.

\section{Case Study \#2: Six Sigma Methodology}

Objective. The next step in the $\mathrm{PhD}$ program was to investigate the effectiveness of another Quality Improvement technique called Six Sigma. The objective of Case Study \#2 was to use Six Sigma methodology to dive deep into the reasons for patient falls to develop an intervention that addresses specific causations of falls with injury. This project was aligned with the Joint Commission's Center for Transforming Healthcare (CTH) to participate with six other hospitals from around the United States using Six Sigma methodology to prevent falls with injury.

Method. Six Sigma methodology (Define, Measure, Analyze, Improve and Control - DMAIC) was used to investigate root causes of the most critical factors such as unassisted toileting and continuously changing patient conditions (cognition, medications).

There were fortnightly conference calls with the $\mathrm{CTH}$ and the other 6 participating hospitals. In the interim week a Small-Core team met as a steering committee to plan and conduct analyses. A twohour meeting was held once a month with the multidisciplinary large team. The following methods were completed in the DMAIC Process:

- $\quad$ Define. During the Define Phase, a charter was developed and the study division was selected. The SIPOC (Supplier, Input, Process, Output, and Customer) method was used to select team members. A Solution Tree was used to collect the Voice-of-the-Customer in order to create a sense of urgency among team members to increase engagement.

\section{Voice of Customer Feedback}

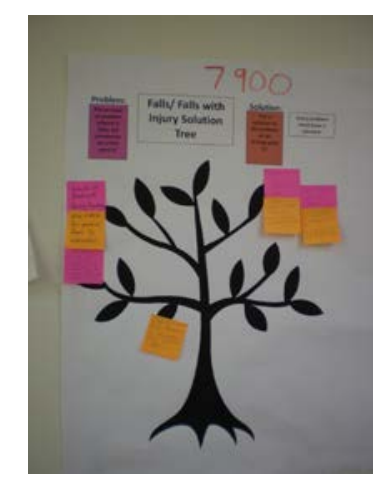

Whole Team Meeting: Affinity Diagram Results:

- Equipment

- Environment

- Call lights

- Communication

- Staffing

- Staff Education \& Awareness

- Patient Assessment

- Patient Education

- Family Education

Figure 1. Examples of Tools used in Six Sigma Define Phase (Solution Tree)

- $\quad$ Measure. A cause-effect matrix was used in the measurement phase to determine the most critical factors related to falls and falls with injury. Fishbone diagrams were then developed for each of the top eight issues to determine the root cause of the problem. A data collection plan was developed to investigate each of the root causes.

- $\quad$ Analyze. In addition to unique measurement issues, each of the hospitals agreed on five common issues and collected data in a similar fashion:

o Call light response time

o Patient behavior at time of fall (reason for getting out of bed)

o Medication management (medications given 24 hours prior to fall compared with patients that did not fall)

o Changes in patient condition 24 hours prior to fall

o Patient activity at time of fall

- Improve. The idea for improvement must be linked to the most critical factors identified during 
the analysis phase (toileting, unassisted falls, and patient's not using call lights). Brainstorming of targeted solutions resulted in an idea called Patient Partnering where a Clinical Expert (Advanced Practice Nurse - APN) would review all patients at risk to fall and mentor staff nurses to partner with patients to heighten the patient's awareness about their increased risk. This intervention empowers the patient to seek assistance from staff when moving about in the room, especially during toileting related activities. The purpose is to encourage patients to call for help and participate in preventing their own falls.

- $\quad$ Control. After a short pilot of the patient partnering process by the APN, the process was taught to all the nurses in the oncology division. Training was conducted during staff meetings and individual coaching was performed as needed. Although momentum was maintained by the APN, the intervention was never fully adopted by the bedside nurses. The nurse will cooperate with the APN in patient partnering, but will not initiate the partnership themselves. The nurses did not adopt the technique due to the perceived additional time requirement.

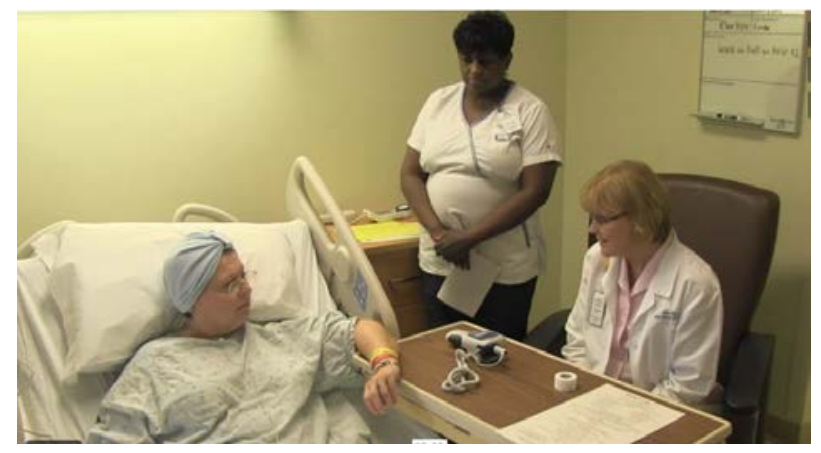

Figure 2. Reenactment of Patient Partnering

Case Study \#2 Results. This intervention was conducted with 87 high fall risk patients where 6\% (5 patients) experienced a fall. Twenty-one of these 87 patients were found to be extremely probable to fall yet only two of these patients fell during their hospital stay.

Patient Partnering related to fall prevention is an innovative approach that empowers the patient to make an informed decision about their own safety. This technique is good nursing care and could be applicable throughout the hospital.

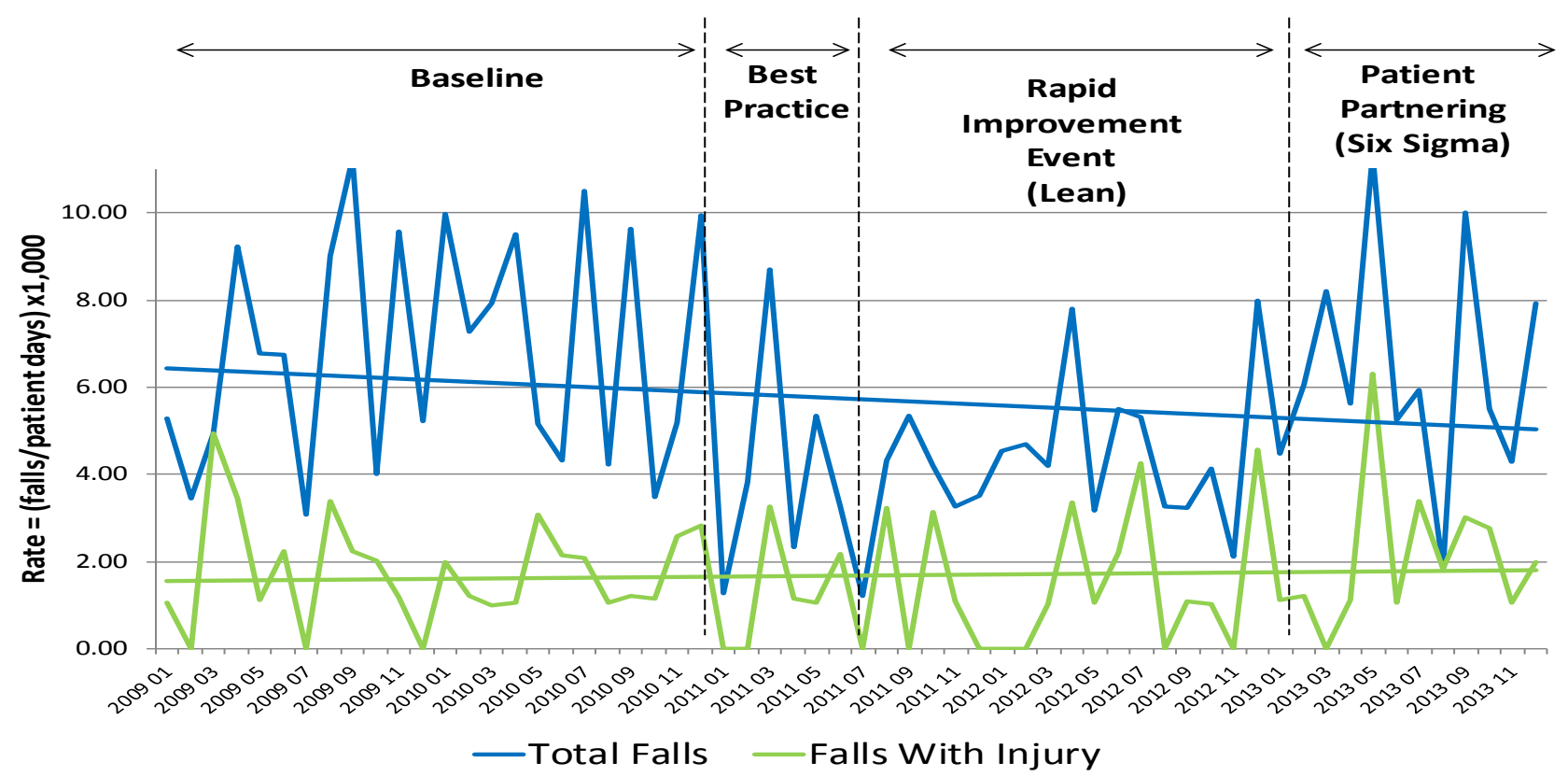

Figure 3. Falls and falls with injury rates in oncology from 2009-2013

Rate $=$ (number of falls/patient days) $\times 1,000$ 


\section{Fall Rate Trends Over Five Years}

Although the 16 months after the Rapid Improvement event (RIE) showed 33\% improvement in total fall rate, the 10 months after the completion of the Six Sigma project, the total falls dropped to only 6\% improvement over baseline (Fig. 3). Another alarming trend is that falls with minor injuries actually increased after Case Study 2 (Six Sigma).

This demands further investigation into the levels of injury that occur from a fall. As shown in Fig. 4, falls with minor injuries have increased but falls with serious injury have decreased (serious includes categories of moderate, major and death). The percent of falls with serious injury have decreased from 10\% in 2010 to 6\% in 2011 and there were no falls with serious injury in 2013.

The fact that falls with minor injuries increased while serious injuries dropped to zero means that more work is needed to understand this issue. A deep understanding of patient and staff perceptions will help to create solutions that can be hardwired and sustained.

\section{INSIGHTS AND REFLECTIONS}

Notes were documented in a journal for more than two years throughout Case Study 1 and 2 (lean and six sigma methodology respectively) to gain an understanding of fall issues. In addition to case studies, the journal included notes from classes, conferences, meetings with advisors and students at Loughborough University and literature reviews. A series of models were also developed in an attempt to gain an overall understanding of the complex topic of falls. Reflection on the journal notes revealed 110 insights that were assembled into nine categories (Fig. 5).

\section{Categories of Insights}

The following nine categories were assembled from 110 insights:

Facilitation/Framework. This category involved understanding the flexibility of tools, strategies, statistics and preparation involved with applying Lean and Six Sigma to fall prevention. Short continuous improvement strategies are most successful in a rapidly changing environment.

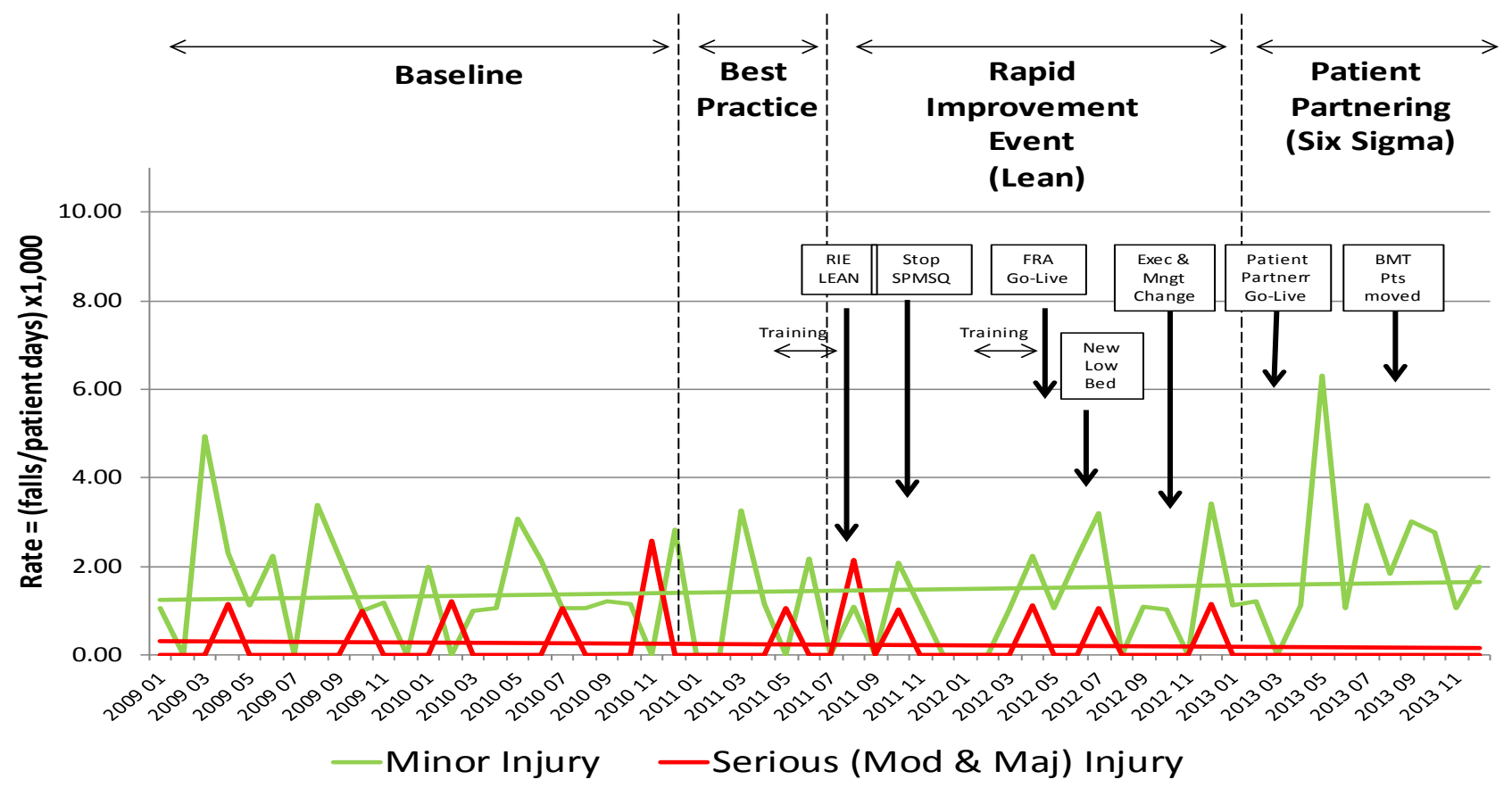

Figure 4. Injury Levels from Falls in oncology from 2009-2013.

Rate $=$ (number of falls/patient days) $\times 1,000$ 
Eleven of the 28 facilitation insights involved framework concepts such as macro verse micro ergonomics.

Complexity. The realization that there is no "silver bullet" or magic answer to prevent falls was the theme throughout this category. There are simply too many intrinsic and extrinsic variables for a one-size-fits-all solution. Three levels of fall risk with 38 possible interventions make algorithms extremely complicated.

Interventions. Dynamic assessment and interventions in a constantly changing environment are the challenging insights in this category. Assessing the patient correctly to match to the most appropriate intervention at the right time was a common theme. Conflicts like privacy verses safety while toileting can make interventions difficult. Some of the insights in this category are intertwined with complexity and critical thinking.

Critical Thinking. A deep understanding of the cause of fall risk and application to each individual patient is critical to gain insight from the intersection of these factors. Nurses must think beyond the checklist mentality to gain a complete understanding of risk factors and bundle together the most appropriate patient safety interventions into a common strategy.
Team \& Pace of Change. Obviously all patient care staff has an important role in fall prevention, but the patient must also be an active participant in order to achieve success. The concept of the pace of change was included in this category because the insight on the length of time required to engage staff had a large impact on team engagement. Small, quick continuous improvements are needed in this high turnover, constantly changing environment.

Patient Partnering. This interactive technique between caregivers and patients is an intervention that was refined during Case Study \#2 to enhance patient engagement and encourage them to participate in their fall intervention process. This may empower a patient and return a sense of control and independence.

World View. This category involves the different perspective that each team member has and how critical it is that everyone understand each other's view point in order to obtain a common goal of patient safety. For example, the oncology population's predisposition to falls (cognition, weakness, diarrhea, etc.) and falls with injury (bone metastasis, bleeding risk, tethers) combined with being accustomed to independence and desire for privacy while toileting warrants consideration when staff encourage patients to use call lights.

Priority and Culture Change. Fall prevention is perceived to be a lower priority than other critical medical issues. A culture change is needed to shift importance of falls to be incorporated into medical decisions and plan of care.

\section{Summary of 110 Insights}

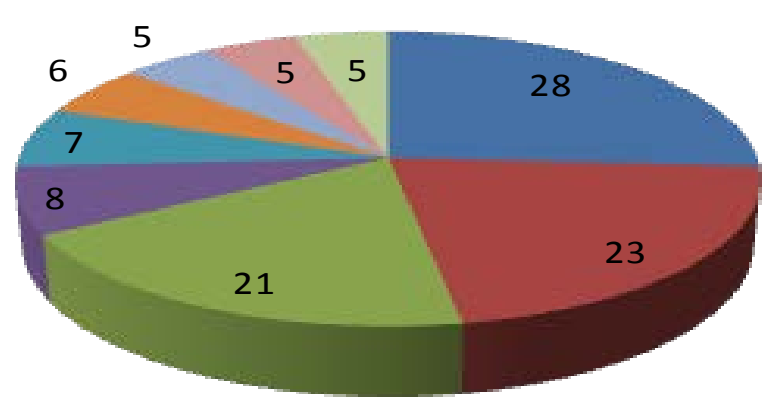

$$
\begin{aligned}
& \text { facilitation } \\
& \text { complexity } \\
& \text { intervention } \\
& \text { critical thinking } \\
& \text { team \& pace of change } \\
& \text { patient partnering } \\
& \text { world view } \\
& \text { priority \& culture change } \\
& \text { environment }
\end{aligned}
$$

Figure 5. Categories of Insights from Case Studies 1 and 2. 
Environment. Most environmental issues in the scope of these insights involve equipment. The design environment such as room layout and flooring is typically thought to be outside of the scope or control of the caregivers and are only mentioned if a hazard is discovered.

\section{DISCUSSION AND CONCLUSION}

The next step in this fall prevention effort is to use Participatory Ergonomics methodology for Case Study \#3 to gain an understanding of patient's perception of risk for falling and injury. Falls are a multi-faceted, complex problem that needs constant vigilance and continuous improvement to sustain patient safety. Anticipating physiologic changes in patient's conditions and implementing interventions before the fall is critical to fall prevention. While well validated screening tools performed thoroughly and accurately can help hospital staff identify patient specific fall risk factors, risk assessment alone does not prevent falls. If the prevention of patient falls is identified as important by leadership and staff at the division level and all are invested in achieving established goals, success can be achieved and sustained.

\section{ACKNOWLEDGMENTS}

The authors would like recognize Cathie Limbaugh (the APN) for her dauntless dedication to keeping patients safe with patient partnering. Without her perseverance a project of this magnitude would not be possible. We would like to express a special thank you to Kathy Rensing and Phyllis Gabbart for their expertise and input and Pat Matt for technical support from the Center for Clinical Excellence at BJC Healthcare.

\section{REFERENCES}

Di Pilla, S., \& Di Pilla, S. (2010). Slip, trip, and fall prevention : a practical handbook (2nd ed.). Boca Raton: CRC Press.

Ulrich, R. S., Zimring, C., Zhu, X., DuBose, J., Seo, H. B., Choi, Y. S., Joseph, A. (2008). A review of the research literature on evidence-based healthcare design. Health Environments Research \& Design Journal, 1(3), 61-125.

Wolf, L., Costantinou, E., Matt, P., \& Schulte, L. (2013). Rapid Improvement Event to Prevent Patient Falls. In V. G. Duffy (Ed.), Advances in human aspects of healthcare (pp. 243-248 p.). Boca Raton: CRC Press.

Wolf, L., Costantinou, E., Limbaugh, C., Rensing, K., Gabbart, P., \& Matt, P. (2013). Fall prevention for inpatient oncology using lean and rapid improvement event techniques. Health Environments Research \& Design Journal, 7(1). 85-101.

Wong, C. A., Recktenwald, A. J., Jones, M. L., Waterman, B. M., Bollini, M. L., \& Dunagan, W. C. (2011). The cost of serious fall-related injuries at three Midwestern hospitals. Jt Comm J Qual Patient Saf, 37(2), 81-87. 